\title{
Therapeutic outcome of various treatment modalities for the management of 34 cases of mandibular unicystic ameloblastoma
}

\author{
Rajib Khadka ${ }^{1}$, Nitesh Chaurasia ${ }^{2}$, Deepak Yadav $^{3}$, Deepak Thakurathi ${ }^{4}$ \\ ${ }^{1}$ Prof. and Director; Sewa Foundation/Cleft program; Vayodha Hospital, ${ }^{2}$ KUMS, Dhulikhel, ${ }^{3}$ UCMS, \\ Bhairawa, ${ }^{4}$ King Edward Medical University, Pakistan
}

\section{Correspondence}

Dr. Deepak Thakurathi

King Edward Medical

University, Pakistan

Email:

deepakthakurathi@hotmail.com

DOI: http://dx.doi.org/10.3126/ jemsn.v14i1.19108

Orcid ID: orcid.org/0000-0003 $-2338-2403$

Article received: Jan $29^{\text {th }} 2018$ Article accepted: Mar $22^{\text {nd }} 2018$

\begin{abstract}
Background \& Objectives: Unicystic ameloblastoma is a challenge, as conservative modalities have high recurrence chances whereas radical modalities have high morbidity for defects and deformity. Enucleation with peripheral ostectomy and Carnoy's solution is an intermediate treatment with less risk of recurrence and good outcome. The objectives of the study was to determine the therapeutic outcome for various treatment modalities for the management of mandibular unicystic ameloblastoma. Materials \& Methods: Retrospective analysis of 34 cases from 2005 to 2014 was done and were analysed in terms of demographic profiles, treatment modalities and its efficacy (recurrence) in 6 years' follow up time. Results: The total number of patients was 34 . The age ranged from 12 years to 28 years with a mean age of 18.82 years. Gender distribution was 21 males $(61.8 \%)$ and 13 females $(38.2 \%)$. The location found was $26(76.5 \%)$ cases in posterior mandibular region and $8(23.5 \%)$ cases in the anterior mandibular region. Size of the lesions was small in $10(29.4 \%)$ cases, medium in $18(52.9 \%)$ cases and large in $6(17.6 \%)$ cases. Perforation of buccal or lingual cortex was present in $6(17.6 \%)$ and no preforation in $28(82.4 \%)$. Treatment modalities done was marsupilisation in $6(17.6 \%)$ cases, enucleation with peripheral ostectomy with caroney solution in $22(64.7 \%)$ cases and resection with safe margin in $6(17.6 \%)$ cases. Recurrence occurred in 8 $(23.5 \%)$ cases and no recurrence in $26(76.5 \%)$ cases. Conclusion: Enucleation with peripheral ostectomy and Carnoy's solution is one of the good treatment modality for unicystic ameloblastoma of the mandible whereas complete resection of the mandible with safe margin has low risk of recurrence in long term follow up.
\end{abstract}

Key words: Ameloblastoma; Carnoy's solution; Curettage; Enucleation; Mandibular resection.

Citation: Khadka R, Chaurasia N, Yadav D, Thakurathi D. Therapeutic outcome of various treatment modalities for the management of 34 cases of mandibular unicystic ameloblastoma. JCMS Nepal. 2018;14 (1):28-31.

\section{INTRODUCTION}

Ameloblastoma is a benign aggressive odontogenic tumor of epithelial origin. ${ }^{1}$ WHO and the International Agency for Research on Cancer, has classified ameloblastoma into Solid/multicystic, Extraosseous/peripheral, Desmoplastic ameloblastoma and Unicystic types. ${ }^{2}$

Unicystic ameloblastoma is the second most common type accounting for 10 to $46 \%$ of intraosseous ameloblastoma. ${ }^{3}$ This type tends to occur in younger population (average 21 years) with growth pattern of $6 \%$ and is associated with impacted tooth and dentigerous cyst; with most common location being the ramus/ molar region of the mandible. ${ }^{4}$

Clinically they present as local swelling, occasional pain with signs of lip numbness. Roentographically they may vary from well-defined unilocular to multilocular radiolucency with 40 to $70 \%$ root resorption. 
Three mechanisms have been proposed for the pathogenesis: i) arising from reduced enamel epithelium, ii) arising from dentigerous or other odontogenic cysts and iii) solid ameloblastoma undergoing cystic degeneration of ameloblastic islands. $^{5}$

Histopathologically three different types of unicystic ameloblastoma has been reported. i)The luminal type is lined by a variable often nondescript epithelium; ii)the intraluminal type shows intraluminal plexiform proliferation of epithelium; whereas iii)intramural type shows invasion of epithelium into the cyst wall in either follicular or plexiform patterns. ${ }^{6}$ The proliferative potential of this type lies between the odontogenic keratocyst and solid ameloblastoma. ${ }^{7}$

Various treatment modalities include marsupialization, enucleation with Carnoy's solution, enucleation with peripheral ostectomy and resection (marginal or segmental). ${ }^{8,9}$

Marsupialization and simple enucleation have shown higher recurrence rate as the pathological tissue is left behind. Resection with safe margin (1 to $1.5 \mathrm{~cm}$ radiographic margin) is a good treatment modality but is associated with various complications such as severe deformity and dysfunction. So to avoid the complications of resection and to reduce the high risk of recurrence with simple enucleation, enucleation has been combined with peripheral ostectomy and with chemical cauterisation which proved to have good therapeutic efficacy and outcome. In peripheral ostectomy, after the enucleation, a small amount of bone is trimmed off the cavity with a large round bur using a coolant. ${ }^{10}$ This is followed by chemical cauterization with Carnoy's solution (absolute alcohol $6 \mathrm{~mL}$, chloroform $3 \mathrm{~mL}$, glacial acetic acid $1 \mathrm{~mL}$, ferric chloride $0.1 \mathrm{gm} / \mathrm{mL}$ ) for about three minutes using cotton applicators, which gave a good prognosis. ${ }^{11,12}$

The aim of our study was to determine the therapeutic outcome for various treatment modalities in the management of mandibular unicystic ameloblastoma.

\section{MATERIALS AND METHODS Materials}

The retrospective analysis was done by Department of oral and maxillofacial surgery at UCMS, Bhairahawa, Nepal.

\section{Sample selection}

Inclusion criteria: Patients diagnosed as unicystic ameloblastoma histopathologically by incisional or excisional biopsy.

Exclusion criteria: Patients exempted from surgery due to lack of fitness

Treatment modality algorithm:

The treatment modalities chosen was as per the radiographic size of the lesion.

$\Rightarrow$ Small $(2 \times 2 \mathrm{~cm})$ were operated with enucleation with caroney solution and peripheral ostectomy.

$\Rightarrow$ Medium $(2 \times 2-4 \times 4)$ size of ameloblastoma were treated with enucleation with peripheral ostectomy and Carnoy's solution, if no perforation of the buccal and lingual cortex was found; resection was done for the cases with perforation

$\Rightarrow$ Large $(>4 \times 4 \mathrm{~cm})$ size was treated with resection with safe margin for perforation and marsupilisation followed by enucleation for nonperforated cases

\section{Data collection and analysis}

Patients with unicystic ameloblastoma diagnosed histolopathologically by incisional or excisional biopsy were collected and analysed. Evaluation was done by taking a brief history and clinical examination and panaromic view of the patients' jaw along with standard photograph. Informed consent was taken from the patient explaining the procedure in detail. Patients were operated with different treatment modalities depending upon the treatment protocol under LA or GA.

Descriptive variables was collected and transferred to statistical package for the social sciences (SPSS) version 20.0.0 computer software and analyzed accordingly. Age, gender and location, size of the lesion, perforation, treatment modalities and recurrence were presented in the form of percentage and bar diagrams.

\section{RESULTS}

The total number of patients was 34. The age ranged from 12 years to 28 years with a mean age of 18.82 years. Gender distribution was 21 males $(61.8 \%)$ and 13 females (38.2\%) .

The location found was $26(76.5 \%)$ cases in distal mandibular region and $8(23.5 \%)$ cases in the proximal mandibular region. Size of the lesions was small in $10(29.4 \%)$ cases, medium in $18(52.9 \%)$ cases and large in $6(17.6 \%)$ cases

Perforation of buccal or lingual cortex was present in $6(17.6 \%)$ and no preforation in $28(82.4 \%)$

Treatment modalities done was marsupilisation in 6 $(17.6 \%)$ cases, enucleation with peripheral ostectomy and Carnoy's solution in $22(64.7 \%)$ cases and resection with safe margin in $6(17.6 \%)$ 
Table 1: Treatment modalities

\begin{tabular}{lll} 
Treatment modalities & Frequency & $\%$ \\
\hline $\begin{array}{l}\text { Marsupialization } \\
\begin{array}{l}\text { Enucleation peripheral } \\
\text { ostectomy and Carnoy's } \\
\text { sol }\end{array}\end{array}$ & 6 & 17.6 \\
\hline Resection & 6 & 64.7 \\
Total & 34 & 17.6 \\
\hline
\end{tabular}

cases. (Table 1) Recurrence occurred in 8 (23.5\%) cases and no recurrence in $26(76.5 \%)$ cases. Preoperative and post operative photographs are shown in figure 1.

\section{DISCUSSION}

Akerman et $\mathrm{al}^{6}$ found the ratio of male to female to be $1.3: 1$. As per our experience, the result was 21 males and 13 females with a ratio of $1.6: 1$, which shows a close variability.

The mean age reported by Akerman etal ${ }^{6}$ was 23.8 years whereas our figures show 18.2 years which reveals that unicystic ameloblastoma occurs in the younger population.
The distribution of the lesion in our study was $76 \%$ in the distal region (angle and ramus) and $24 \%$ in the proximal region (parasymphysis and body region). Olaintan et $\mathrm{al}^{13}$ reported UA affecting the symphysis with extension to the canine region $(23.8 \%)$; affecting the premolar and molar region $(28.6 \%)$; affecting the premolar, molar and the angle of the mandible (23.8\%); affecting the angle and the ascending ramus (9.5\%); and associated with all involvement posteriorly $(14.3 \%)$. Both the studies show that distal part of mandible was more prevalent with the pathology.

Various treatment modalities are mentioned in the literature. We used marsupialization for the large lesions, enucleation with Carnoy's solution for medium and large lesions and resection for the lesions with cortical perforation. Decompression and dredging treatment modalities have also been discussed in the literature. ${ }^{14,15}$

Lau and Samman et $\mathrm{al}^{8}$ found the recurrence rates for unicystic ameloblastomas to be $3.6 \%$ after resection; $30.5 \%$ after enucleation alone; $16 \%$ after enucleation followed by application of Carnoy's solution; and $18 \%$ after marsupialization . Our recurrence rate was $23.5 \%$

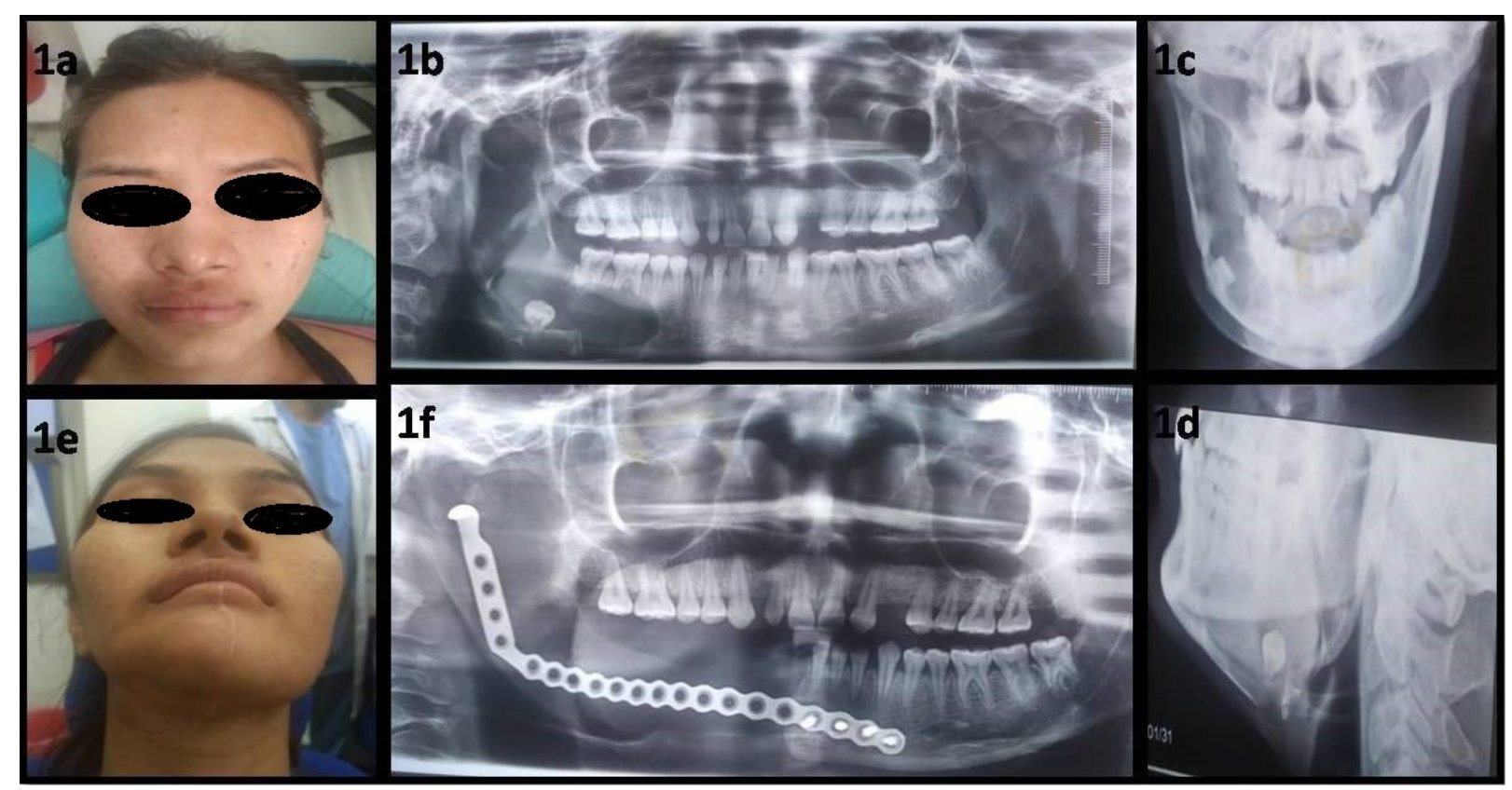

Fig 1a: Preoperative photograph.

Fig 1b: OPG showing a large multilocular radiolucency associated with impacted mandibular $3^{\text {rd }}$ molar.

Fig 1c: PA view showing the same multilocular radiolucency on right mandibular region.

Fig 1d: Lateral oblique view showing the same multilocular radiolucency on right mandibular region with impacted $3^{\text {rd }}$ molar.

Fig 1e: Post operative photograph with sutures in place.

Fig 1f: Post operative OPG showing the excision of the lesion with the placement of arch bars. 
after overall treatment modalities. Further, recurrence rate after marsupialization for large defect was $60 \%$ whereas after enucleation and peripheral ostectomy for small defect was $10 \%$ and for medium defects was $41 \%$. We found no recurrence after complete resection with safe margin. The variance in the recurrence rate may be due to the prudent decision making in the differential treatment modalities.

\section{CONCLUSION}

Enucleation with peripheral ostectomy and Carnoy's solution is one of the good treatment modality for small and medium unicystic ameloblastoma of the mandible which is more of a conservative approach, however complete resection of the mandible with safe margin is more beneficial to the patient and less cumbersome to the operator due to low risk of recurrence in long term follow up.

\section{Funding}

None

\section{Conflict of Interest Statement:}

\section{None Declared}

\section{REFERENCES}

1. Regezi JA, Sciubba JJ, Jordan RC. Oral pathology: clinical pathologic correlations. Elsevier Health Sciences; 2016 Feb 25.

2. Wright JM, Odell EW, Speight PM, Takata T. Odontogenic tumors, WHO 2005: where do we go from here?Head Neck Pathol. $2014 \quad$ Dec 1;8(4):373-82. https://doi.org/10.1007/s12105-014-0585-x. PMID: 25409849.

3. Neville BW, Damm DD, Chi AC, Allen CM. Oral and maxillofacial pathology. Elsevier Health Sciences; 2015.

4. Kessler HP. Intraosseous ameloblastoma. Oral Maxillofac Surg Clin North Am. 2004 Aug;16(3):309-22. https:// doi.org/10.1016/j.coms.2004.03.001. PMID: 18088733 .

5. Reichart PA, Philipsen HP. Odontogenic tumors and allied lesions. Quintessence Pub.; 2004 Jan.

6. Ackermann GL, Altini M, Shear M. The unicystic ameloblastoma: a clinicopathological study of 57 cases. J Oral Pathol Med. 1988 Nov 1;17(9-10):541-6. https:// doi.org/10.1111/j.1600-0714.1988.tb01331.x.

7. Miloro M, Ghali GE, Larsen P, Waite P. Peterson's principles of oral and maxillofacial surgery. PMPH-USA; 2004.

8. Lau SL, Samman N. Recurrence related to treatment modalities of unicystic ameloblastoma: a systematic review. Int J Oral Maxillofac Surg. 2006 Aug 31;35(8):681 -90 .

https://doi.org/10.1016/j.ijom.2006.02.016.

PMID: 16782308.

9. Sachs SA. Surgical excision with peripheral ostectomy: A definitive, yet conservative, approach to the surgical management of ameloblastoma. J Oral Maxillofac Surg. 2006 Mar 1;64(3):476-83. https://doi.org/10.1016/j.joms.2005.12.001. PMID: 16487812.

10. Shi S, Liu Y, Shan Y, Fu T, Zhao S. Enucleation combined with peripheral ostectomy: its role in the management of large cystic ameloblastomas of the mandible.
Craniomaxillofac Surg. 2014 Dec 31;42(8):1659-63. https://doi.org/10.1016/j.jcms.2014.05.009. PMID 24969769

11. Lee PK, Samman N, Ng IO. Unicystic ameloblastomause of Carnoy's solution after enucleation. Int J Oral Maxillofac Surg. 2004 Apr 1;33(3):263-7. https:// doi.org/10.1006/ijom.2003.0496 PMID: 15290793.

12. Rao K, Kumar S. The use of enucleation and chemical cauterization (Carnoy's) in the management of odontogenic keratocyst of the jaws. Indian J Otolaryngol Head Neck Surg. 2014 Jan 1;66(1):8-12. https://doi.org/10.1007/s12070-012-0523-8. PMID: 24605293

13. Olaitan AA, Adekeye EO. Unicystic ameloblastoma of the mandible: a long-term follow-up. J Oral Maxillofac Surg . 1997;55(4):345-8.

https://doi.org/10.1016/S0278-2391(97)90122-1.

14. Park HS, Song IS, Seo BM, Lee JH, Kim MJ. The effectiveness of decompression for patients with dentigerous cysts, keratocystic odontogenic tumors, and unicystic ameloblastoma. J Korean Assoc Oral Maxillofac Surg. 2014 Dec 1;40(6):260-5. https://doi.org/10.5125/ jkaoms.2014.40.6.260. PMID: 25551089 .

15. Kawamura M. Dredging method; a new approach for the treatment of ameloblastoma. Asian J Oral Maxillofac. Surg. 1991;3:81-8. 